\title{
Influence saisonnière des ressources alimentaires sur la distribution des touracos (Musophagidae) dans quelques milieux naturels du Centre et du Sud-est de la Côte d'Ivoire.
}

Okon Modeste OKON, Kouakou Pierre KOUADIO, Ekoun Michaël KONAN et Hilaire K. YAOKOKORE-BEIBRO*

URF de Biologie de la Conservation et Gestion de la Faune, UFR Biosciences de l'Université Félix HouphouëtBoigny, Abidjan-Cocody, 22 BP 582 Abidjan 22, Côte d'Ivoire.

*Auteur correspondant. E-mail: hyaokokore@yahoo.fr, Tel : +22507634265

Original submitted in on $23^{\text {rd }}$ April 2019. Published online at www.m.elewa.org/journals/ on $31^{\text {st }}$ July 2019 https://dx.doi.org/10.4314/jab.v139i1.4

\begin{abstract}
RESUME
Objectifs: Cette étude vise à étudier l'influence des ressources alimentaires sur la distribution des touracos dans quatre milieux naturels que sont les parcs nationaux du Banco et d'Azagny (PNA), la Réserve Scientifique de Lamto et la Forêt Classée de Yapo-Abbé afin de dégager son importance dans la conservation des touracos.

Méthodologie et résultats: Un inventaire saisonnier des touracos dans chaque site a été effectué d'août 2015 à juillet 2016 à l'aide de la méthode des indices ponctuels d'abondance (IPA) (Bibby et al., 1998). Parallèlement, par observation directe, les espèces végétales et leurs organes exploités par les touracos ont été inventoriés. De même, la fréquence d'occurrence des touracos par rapport à ces ressources alimentaires a été calculée afin de déterminer l'influence des ressources alimentaires sur leur distribution. Les résultats indiquent que l'abondance des touracos a varié selon les saisons. La distribution des touracos est liée à la disponibilité des ressources alimentaires dans les milieux naturels.

Conclusion et application des résultats : Cette étude contribue à une meilleure connaissance de l'écologie alimentaire des espèces des touracos et fournir des données sur leur distribution dans les milieux naturels. Ces données pourront contribuer à la mise en place d'une politique de préservation de ces espèces caractéristiques des forêts africaines dans le cadre de la conservation de la biodiversité.
\end{abstract}

Mots clés: Ressources alimentaires, effet saison, conservation et biodiversité.

\begin{abstract}
Objectives: This study aims to study the influence of food resources on the distribution of the turacos in four natural environments, namely the Banco and Azagny national parks, the Lamto Scientific Reserve and the Yapo-Abbé classified forest in order to identify its importance in the conservation of turacos.

Methodology and results: A seasonal inventory of the turacos at each site was carried out from August 2015 to July 2016 using the Point abundance index (PAl) method (Bibby et al., 1998). At the same time, by direct observation, the plant species and their organs exploited by the turacos have been inventoried. Similarly, the frequency of occurrence of the turacos in relation to these food resources was calculated in order to determine the influence of the food resources on their distribution. The results indicate that the
\end{abstract}


abundance of turacos varied seasonally. The distribution of the turacos is linked to the availability of food resources in natural environments

Conclusions and application of findings: This study contributes to a better knowledge of the food ecology of turacos species and to provide data on their distribution in the natural environments. These data can contribute to the establishment of a policy of preservation of these characteristic species of african forests as part of the conservation of biodiversity

Keywords: Food resources, season effect, conservation and biodiversity.

\section{INTRODUCTION}

Les milieux naturels regorgent de nombreux écosystèmes qui offrent aux diverses espèces qui les occupent des habitats nécessaires à leur survie. Ces écosystèmes constituent de fait des habitats remarquables de la faune sauvage (Mbété et al., 2010). Au sein d'un écosystème, les espèces ne se répartissent pas de façon aléatoire mais leur distribution est liée à la satisfaction de leurs besoins écologiques. Divers facteurs notamment les ressources alimentaires conditionnent la présence d'une espèce dans un milieu (Keitt et al., 2002). Les touracos (Musophagidae) sont des espèces arboricoles et frugivores qui sont importantes pour la biodiversité, du fait de leur rôle dans la dispersion des graines

\section{MATERIEL ET METHODES}

Milieu d'étude : Cette étude a été réalisée dans les Parcs nationaux du Banco (PNB) et d'Azagny (PNA), la Réserve scientifique de Lamto (RSL) et la Forêt classée de Yapo-Abbé (Figure 1). Le Parc National du Banco (PNB) avec 3474 hectares, est situé à Abidjan. II est sous l'influence d'un climat subéquatorial à quatre saisons et a une végétation de type forêt dense sempervirente (Lauginie, 2007). L'avifaune du PNB est riche de 203 espèces (Kouadio et al., 2014). Le Parc National d'Azagny (PNA) s'étend sur 19400 hectares et est situé à Grand-Lahou. Son climat est de type subéquatorial humide à quatre saisons (Lauginie, 2007). C'est une mosaïque de formations végétales composée de forêts sempervirentes, de forêts secondaires, de jachères, de marécages, de savanes
(Sun et Moermond, 1997). Malgré cette importance, l'écologie alimentaire des touracos dans les milieux naturels est peu documentée (Borghesio, 1997). Les études ornithologiques en Côte d'Ivoire se sont limitées sur leur diversité sans toutefois faire mention des facteurs de leur distribution dans les milieux naturels (Thiollay, 1985; Yaokokoré-Beibro, 2001). Cependant, la connaissance de l'écologie alimentaire des touracos revêt d'une importance capitale dans le cadre de leur conservation. Ainsi, pour contribuer à une meilleure connaissance de l'influence des ressources alimentaires sur la distribution des touracos, cette étude a été initiée.

et de fourrés littoraux (Gnagbo, 2016). Son avifaune compte 162 espèces (Lauginie, 2007). La forêt classée de Yapo-Abbé (FCYA) couvre une superficie de 24592 hectares et est à $55 \mathrm{~km}$ d'Abidjan (SODEFOR ,1999). Son climat est de type subéquatorial à quatre saisons (SODEXAM, 2013). C'est une forêt dense sempervirente avec 225 espèces d'oiseaux (Demey et Fishpool, 1994). La Réserve Scientifique de Lamto (RSL) s'étend sur 2700 hectares. Elle est située à Taabo et influencée par un climat de type tropical humide à quatre saisons (Lauginie, 2007). C'est une mosaïque forêts-savanes, constituée de galeries forestières, de forêt dense, de savanes préforestières à rôniers (Deniveau, 1976). Son avifaune est diversifiée et riche de 400 espèces (Demey et Fishpool, 1991). 


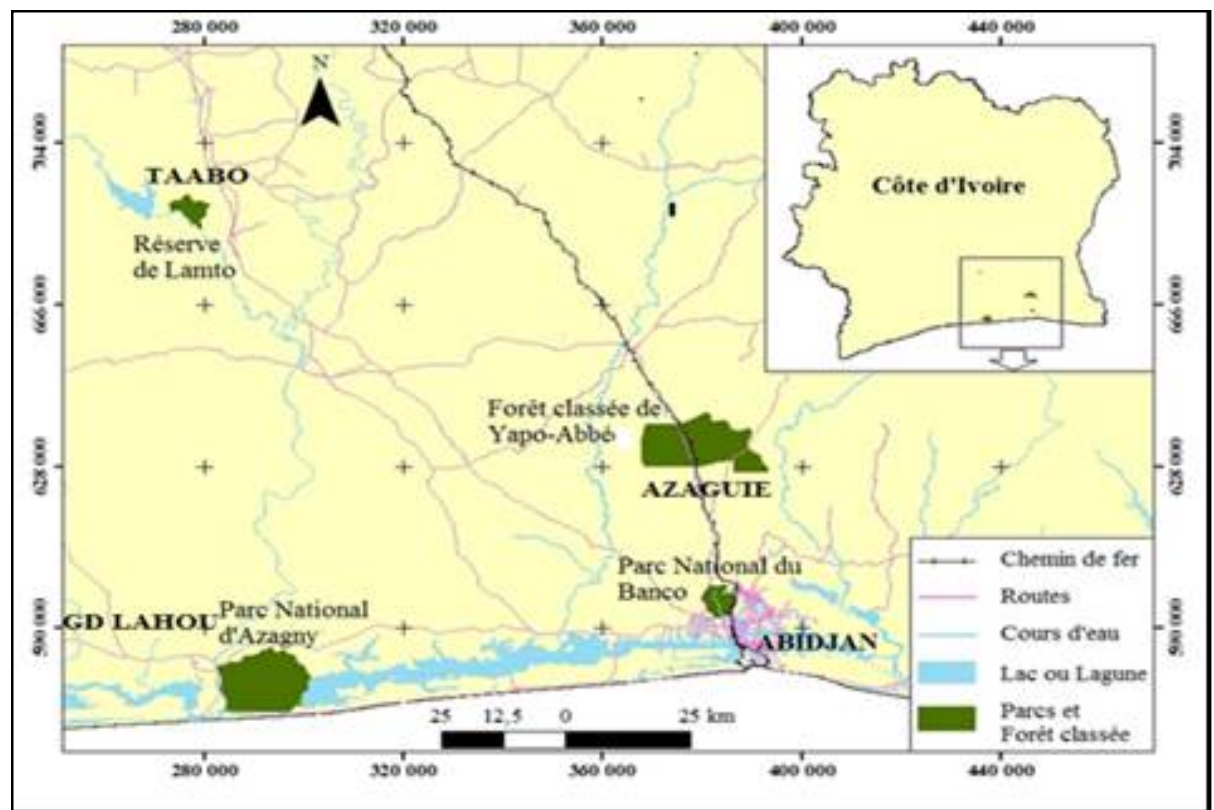

Figure 1 : Localisation de l'ensemble des sites échantillonnés au cours de l'étude

Matériel : Pour la réalisation de cette étude, une paire de jumelles (10 x 50 BRESSER Hunter) a été utilisée pour observer les oiseaux. Un guide d'identification (Borrow et Demey, 2012) a servi à l'identification des oiseaux. Pour les prises de vue, un appareil photographique numérique (SONY DSC-HX 300 (X50)) a été utilisé et un GPS (GPS MAP $60 \mathrm{C}$ ) a permis de géolocaliser les stations d'observation.

Méthode de collecte des données

Méthode d'échantillonnage des touracos: L'étude s'est déroulée d'août 2015 à juillet 2016 dans quatre sites (Figure 1). Dans chacun de ces sites, six habitats ont été identifiés sur la base de leurs caractéristiques écologiques. La méthode des relevés utilisée est celle des points d'écoute couplée des indices ponctuels d'abondance (IPA) (Bibby et al., 1998). Dans chaque habitat, sept points d'écoute ont été installés le long des pistes d'aménagement. Chaque point d'écoute a été repéré par un GPS et marqué à l'aide d'un ruban rouge. La distance comprise entre deux points d'écoute consécutifs a été de $300 \mathrm{~m}$ (Bibby et al., 1998). Cette distance a été choisie afin d'éviter les doublons (Yaokokoré-Beibro, 2001). Les inventaires sont faits de façon journalière, de $6 \mathrm{~h}$ à $12 \mathrm{~h}$ puis de $14 \mathrm{~h} 30$ à $18 \mathrm{~h}$. Le temps mis dans chaque point d'écoute est de 20 minutes au cours duquel les espèces de touracos et leur abondance ont été notées. Les relevés ont été faits par saison dans chaque site.

Méthode de détermination des ressources alimentaires des touracos: Pour l'étude des ressources alimentaires des touracos, une observation directe de toutes les espèces végétales dont les organes ont été consommés par les touracos a été faite.

Méthodes d'analyse des données: Les données recueillies ont permis de calculer la fréquence relative $(\mathrm{Fr}=(\mathrm{ni} / \mathrm{N}) \times 100)$ des touracos au cours des saisons, où ni représente l'effectif d'une espèce i et $\mathrm{N}$ l'effectif total des touracos. Le test non- paramétrique de Kruskal-wallis réalisé avec le logiciel Statistica 7.1, a été utilisé pour mesurer le degré de variabilité saisonnière de l'abondance des touracos. II a été calculé les fréquences d'occurrence $(\mathrm{Fc}=(\mathrm{Si} / \mathrm{St}) \times 100)$ des touracos par rapport aux espèces végétales et aux organes consommés. Aussi, des tests de corrélation de Spearman réalisés à l'aide du logiciel Statistica 7.1, ont été établis entre l'abondance des touracos et les espèces végétales ainsi qu'avec les organes. Une analyse canonique de Redondance RDA au moyen du logiciel Canoco 4.5 a été aussi réalisée pour évaluer l'influence des espèces végétales sur la distribution des touracos. 
RESULTATS

Variation saisonnière de l'abondance des touracos :
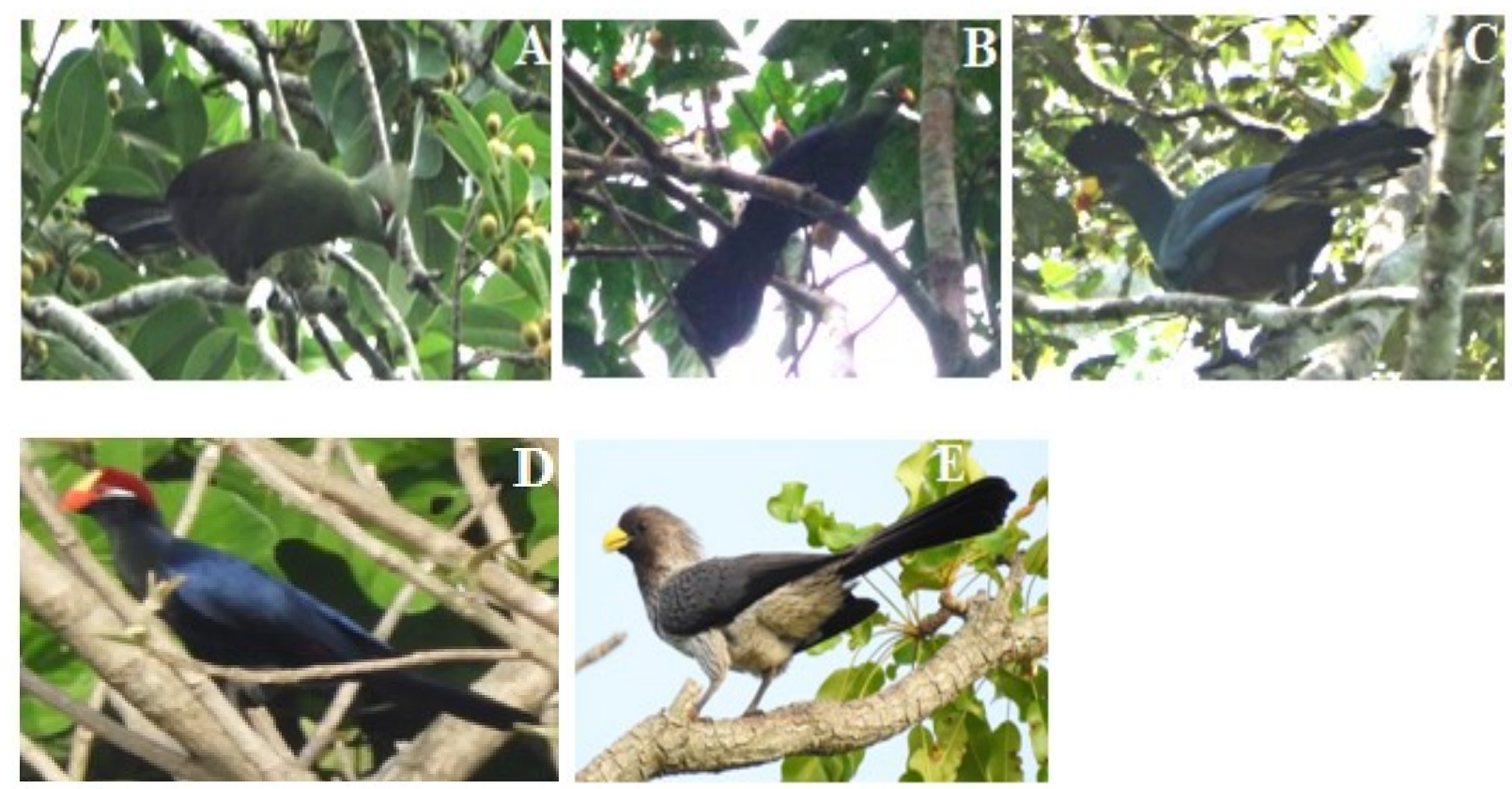

Figure 2 : Photographies des espèces de touracos inventoriées dans l'ensemble des sites

A : Touraco vert Tauraco persa (Linné, 1758), B : Touraco à gros bec Tauraco macrorhynchus (Fraser, 1839), C: Touraco géant Corythaeola cristata (Vieillot, 1816), D: Touraco violet Musophaga violacea Isert, 1788, E: Touraco gris Crinifer piscator (Boddaert, 1783)

Au total, cinq espèces de touracos (Figure 2) avec 939 individus ont été inventoriées dans l'ensemble des sites. L'abondance de ceux-ci est variable selon les saisons. La petite saison sèche (PSS) compte le plus d'individus avec 313 individus ( $\mathrm{Fr}=33,34 \%)$. Par contre, la grande saison sèche (GSS) a le plus faible nombre d'individus avec 162 individus ( $\mathrm{Fr}=17,25 \%)$ (Figure 3). Aussi, L'abondance des touracos selon les saisons diffère significativement (Test de Kruskal wallis, $p=0,012$ ) (Tableau 1).

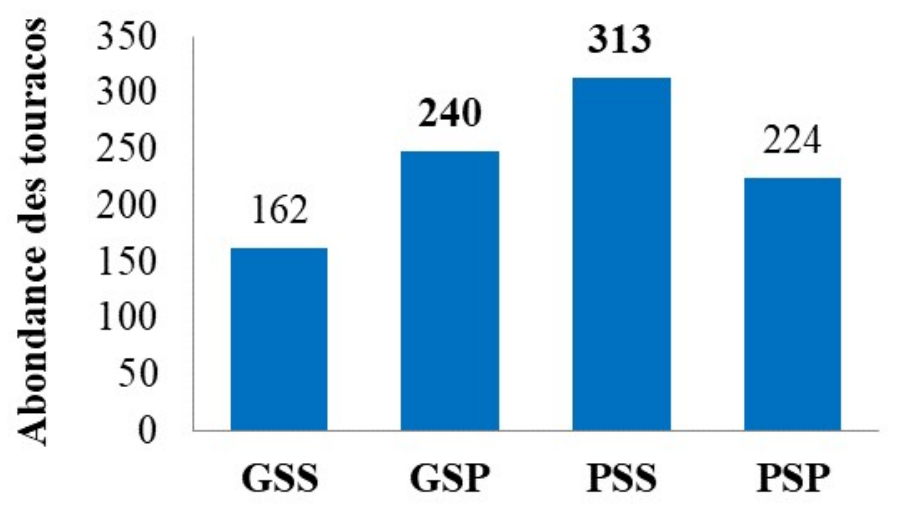

Saisons

Figure 3 : Variation saisonnière de l'abondance relative des touracos

GSS : Grande saison sèche GSP : Grande saison des pluies PSS : Petite saison sèche ; PSP : Petite saison des pluies 
Tableau 1: Significativité de l'abondance des touracos selon les saisons

\begin{tabular}{l|l|l|l|l}
\hline & GSS & \multicolumn{1}{|c|}{ GSP } & \multicolumn{1}{c}{ PSS } & \multicolumn{1}{c}{ PSP } \\
\hline GSS & \multicolumn{1}{|c|}{-} & $0,010756^{*}$ & $0,012966^{*}$ & 0,527740 \\
GSP & $0,010756^{*}$ & 1,000000 & 1,000000 \\
PSS & $0,012967^{*}$ & 1,000000 & 1,0000000 & 1,0000 \\
PSP & 0,527740 & 1,000000 & 1,00000 & - \\
\hline
\end{tabular}

*différence significative $p<0,05$ (Test de Kruskal wallis)

GSS : Grande saison sèche GSP : Grande saison des pluies PSS : Petite saison sèche ; PSP : Petite saison des pluies

Influence des ressources alimentaires sur la distribution des touracos

Ressources alimentaires des touracos: Dans l'ensemble des sites, les touracos ont consommé les organes de 39 espèces végétales appartenant à 20 familles (Tableau 2). La fréquence d'occurrence des touracos en activité de nourrissage est élevée pour
Musanga cecropioides $(9,72 \%)$ et Dialium guineense $(2,78 \%)$ qui sont les deux espèces végétales dont les organes ont été les plus consommés (Tableau 2). La fréquence d'occurrence des touracos sur les organes végétaux varie d'un organe à l'autre. Cette fréquence est forte pour les fruits $(25,69 \%)$ et faible pour les fleurs $(0,69 \%)$ (Figure 4 ).

Tableau 2: Fréquence d'occurrence des espèces de touracos en activité de nourrissage par rapport aux espèces végétales dans l'ensemble des sites.

\begin{tabular}{|c|c|c|}
\hline Familles & Espèces végétales & $\begin{array}{l}\text { Fréquence d'occurrence }(\mathrm{Fc}) \text { en } \\
\%\end{array}$ \\
\hline \multirow[t]{2}{*}{ Anacardiaceae } & Lannea welwitschii (Hiern) Engl. & 0,35 \\
\hline & Spondias mombin Jacquin & 0,69 \\
\hline Annonaceae & Cleistopholis patens Benth \& Diels & 0,69 \\
\hline Araliaceae & Cussonia barteri Seem. & 0,69 \\
\hline \multirow[t]{3}{*}{ Arecaceae } & Borassus aethiopum Martius & 0,35 \\
\hline & Elaeis guinensis Jacquin & 1,04 \\
\hline & Phoenix reclinata Jacquin & 0,35 \\
\hline \multirow[t]{2}{*}{ Burseraceae } & Canarium schweinfurthii Engl. & 0,35 \\
\hline & Dacryodes klaineana (Pierre) H.J.Lam & 0,35 \\
\hline Cannabaceae & Celtis zenkeri Engl. & 1,39 \\
\hline Chrysobalanaceae & Parinari glabra Oliv. & 0,35 \\
\hline Ebenaceae & Diospyros mespiliformis Horht. Ex A.DC. & 0,35 \\
\hline Euphorbiaceae & Discoglypremna caloneura Prain & 1,04 \\
\hline \multirow[t]{5}{*}{ Fabaceae } & Dialium dinklagei Harms & 1,39 \\
\hline & Dialium guineense Willd & 2,78 \\
\hline & Piptadeniastrum africanum (Hook. F) & 1,04 \\
\hline & Pterocarpus erinaceus Poir & 0,35 \\
\hline & Afzelia africana Sn. \& Pers. & 0,35 \\
\hline Lecythidaceae & Scytopetalum tieghemii Hutch. \& Dalziel & 1,39 \\
\hline \multirow[t]{3}{*}{ Meliaceae } & Azadirachta indica A. Juss. & 1,04 \\
\hline & Guarea cedrata A.Chev. & 1,74 \\
\hline & Turraeanthus africanus Welw & 0,35 \\
\hline \multirow[t]{2}{*}{ Phyllanthaceae } & Bridelia ferruginea Benth. & 0,35 \\
\hline & Uapaca guineensis Muell. & 0,35 \\
\hline \multirow[t]{5}{*}{ Moraceae } & Ficus dicranostyla Mildbr. & 0,35 \\
\hline & Ficus djalonensis A.chev. & 0,69 \\
\hline & Ficus exasperata Vahl & 0,35 \\
\hline & Ficus goliath A.chev. & 0,69 \\
\hline & Ficus mucuso Welw & 0,69 \\
\hline
\end{tabular}




\begin{tabular}{l|l|c}
\hline Familles & Espèces végétales & $\begin{array}{l}\text { Fréquence d'occurrence (Fc) en } \\
\%\end{array}$ \\
\hline & Ficus ovata Vahl & 0,35 \\
Rhizophoraceae & Morus mesozygia Stapf & 0,69 \\
Rubiaceae & Cassipourea hiotou Auibrev. \& Pellegr & 1,39 \\
& Crossopteryx febrifuga Benth. & 1,04 \\
Sapotaceae & Morinda lucida Benth. & 0,69 \\
& Afrosersalisia afzelii A.chev. & 0,35 \\
Sterculiaceae & Malacantha heudelotiana Pierre & 0,35 \\
Strombosiaceae & Cola gigantea A .chev. & 0,35 \\
Urticaceae & Strombosia glaucescens Engl. & 0,35 \\
\hline
\end{tabular}

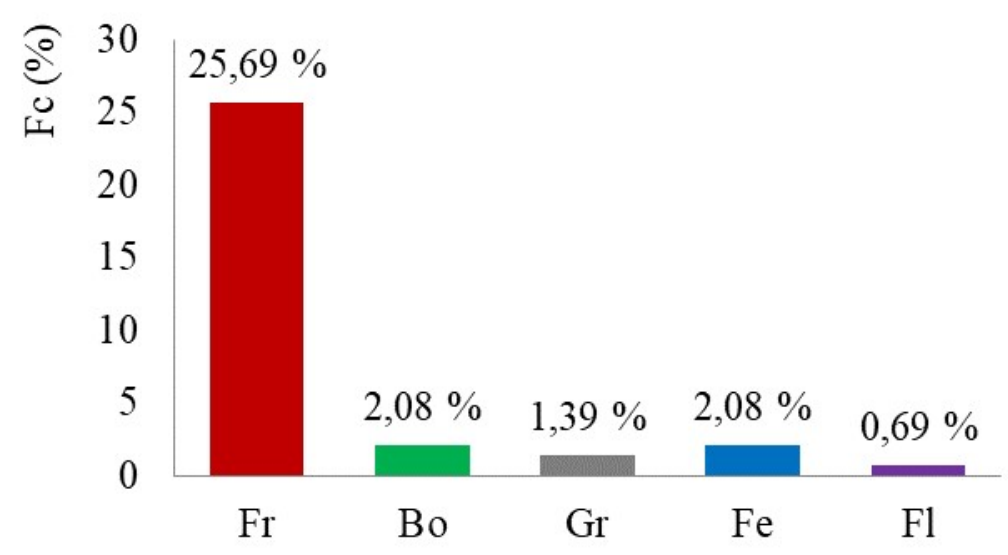

\section{Organes végétaux}

Figure 4 : Fréquence d'occurrence $(\%)$ des touracos sur les organes végétaux

$\mathrm{Fr}=$ fruits, $\mathrm{Bo}=$ bourgeons $; \mathrm{Gr}=$ graines $; \mathrm{Fe}=$ feuilles $; \mathrm{Fl}=$ fleurs, $\mathrm{FC}=$ Fréquence d'occurrence $(\%)$

Variation saisonnière des ressources alimentaires : L'exploitation des espèces végétales par les touracos dans l'ensemble des sites varie d'une saison à l'autre. Ainsi, Musanga cecropioides (Urticaceae), Cassipourea hiotou (Rhizophoraceae), Elaeis guinensis (Arecaceae), Dialium guineense (Fabaceae) ont été exploitées par les touracos presque régulièrement toutes les saisons de l'année. Par contre, les autres espèces végétales ont été exploitées de façon ponctuelle au cours d'une seule saison (Figure 5). Toutefois, le test de Kruskal wallis effectué indique qu'il y a une différence significative de l'exploitation des espèces végétales au cours des saisons $(P<0,05)$. 


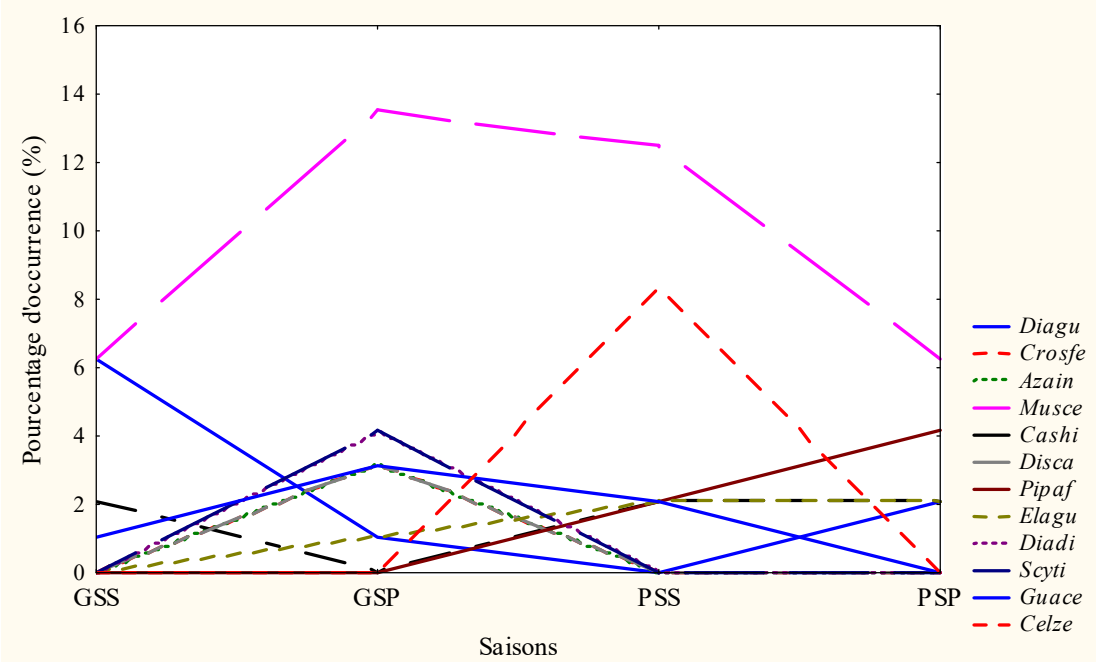

Figure 5 : Variation saisonnière de la fréquence d'occurrence des touracos par rapport aux espèces végétales dans l'ensemble des sites.

Diagu: Dialium guineense ; Crosfe : Crossopteryx febrifuga; Azain : Azadirachta indica; Musce : Musanga cecropioides; Cashi : Cassipourea hiotou; Disca : Discoglypremna caloneura ; Pipaf : Piptadeniastrum africanum; Elagu : Elaeis guinensis; Diadi : Dialium dinklagei; Scyti : Scytopetalum tieghemii; Guace : Guarea cedrata; Celze : Celtis zenkeri

GSS : Grande saison sèche GSP : Grande saison des pluies PSS : Petite saison sèche PSP : Petite saison des pluies

Influence des espèces végétales et leurs organes sur les touracos: Le test de corrélation réalisé avec les 12 espèces végétales les plus exploitées par les touracos, indique une corrélation positive entre l'abondance des touracos et la fréquence d'exploitation de Celtis zenkeri (Cannabaceae) avec $r=0,63 ; p>$ 0,05 . De même, une forte corrélation positive existe entre l'abondance des touracos et la fréquence d'exploitation de Dialium guineense (Fabaceae) avec $r$
$=0,77 ; p>0,05$. Par contre cette corrélation est négative entre l'abondance des touracos et la fréquence d'exploitation de Dialium dinklagei avec $r=$ 0,$77 ; p>0,05$ (Tableau 3). Les coefficients de Spearman indiquent une forte corrélation positive entre l'abondance des touracos et la fréquence d'exploitation des fruits et des bourgeons avec respectivement $r=$ 0,$60 ; p>0,05$ et $r=0,63 ; p>0,05$. (Tableau 4).

Tableau 3 : Coefficient de corrélation de Spearman entre les espèces végétales et l'abondance des touracos dans l'ensemble des sites

\begin{tabular}{|c|c|c|}
\hline Espèces & $\mathbf{r}$ & $p$ \\
\hline Celze & 0,63 & 0,37 \\
\hline Disca & $-0,26$ & 0,74 \\
\hline Diadi & $-0,77$ & 0,23 \\
\hline Diagu & 0,77 & 0,23 \\
\hline Pipaf & 0,11 & 0,89 \\
\hline Scyti & $-0,77$ & 0,23 \\
\hline Azain & 0,77 & 0,23 \\
\hline Guace & 0,26 & 0,74 \\
\hline Ficdj & 0,77 & 0,23 \\
\hline Cashi & $-0,26$ & 0,74 \\
\hline Crosfe & 0,77 & 0,23 \\
\hline Musce & $-0,40$ & 0,60 \\
\hline
\end{tabular}

Celze : Celtis zenkeri ; Disca: Discoglypremna caloneura; Diadi : Dialium dinklagei; Diagu: Dialium guineense ; Pipaf: Piptadeniastrum africanum; Scyti : Scytopetalum tieghemii; Azain : Azadirachta indica; Guace : Guarea cedrata ; Elagu : Elaeis guinensis; Cashi : Cassipourea hiotou; Crosfe : Crossopteryx febrifuga; Musce : Musanga cecropioides. 
Tableau 4 : Coefficient de corrélation de Spearman entre les organes végétaux et l'abondance des touracos dans l'ensemble des sites

\begin{tabular}{lc|c|c}
\hline Organes & $\mathbf{r}$ & $\mathbf{p}$ \\
\hline $\mathrm{Fr}$ & 0,60 & 0,40 \\
$\mathrm{Bo}$ & 0,63 & 0,37 \\
$\mathrm{Gr}$ & $-0,26$ & 0,74 \\
$\mathrm{Fe}$ & 0,95 & 0,05 \\
$\mathrm{FI}$ & 0,89 & 0,11 \\
\hline
\end{tabular}

Fr : fruits ; Bo : bourgeons ; Gr : graines ; Fe : feuilles ; FI : fleurs

Relation entre les espèces de touracos et les espèces végétales selon les sites: Une analyse canonique des Redondances (RDA) a été utilisée pour mettre en évidence la corrélation entre les espèces de touracos et les espèces végétales. Le parc national du Banco (PNB) et la forêt classée de Yapo-Abbé (FCYA) sont corrélés positivement l'axe 1. Tauraco macrorhynchus est fortement associé à ces deux sites au sein desquels l'espèce a exploité les organes de cinq espèces végétales. Par ailleurs, dans le PNB, $T$. macrorhynchus a consommé beaucoup plus les fruits de Musanga cecropioides (Urticaceae) et de Guarea cedrata (Meliaceae) ainsi que les bourgeons de Piptadeniastrum africanum (Fabaceae). De même, dans la FCYA, l'espèce a plus consommé les fruits de Dialium dinklagei (Fabaceae) et de Scytopetalum tieghemii (Lecythidaceae). Par contre, la Réserve scientifique de Lamto (RSL) et le Parc national d'Azagny (PNA) sont corrélés négativement à l'axe 1. Tauraco persa, Crinifer piscator, Corythaeola cristata et Musophaga violacea sont associées à ces deux sites. Dans ces sites, les touracos ont exploité les organes de sept espèces végétales. Par ailleurs, dans la RSL, les espèces de touracos ont consommé les fruits de Dialium guineense (Fabaceae), Celtis zenkeri (Cannabaceae), Azadirachta indica (Meliaceae) et Crossopteryx febrifuga (Rubiaceae). De même, dans le PNA, Crinifer piscator a consommé les fruits de Cassipourea hiotou (Rhizophoraceae) et Elaeis guinensis (Arecaceae) alors que Corythaeola cristata a consommé les fruits de Discoglypremna caloneura (Euphorbiaceae) (Figure 6). 


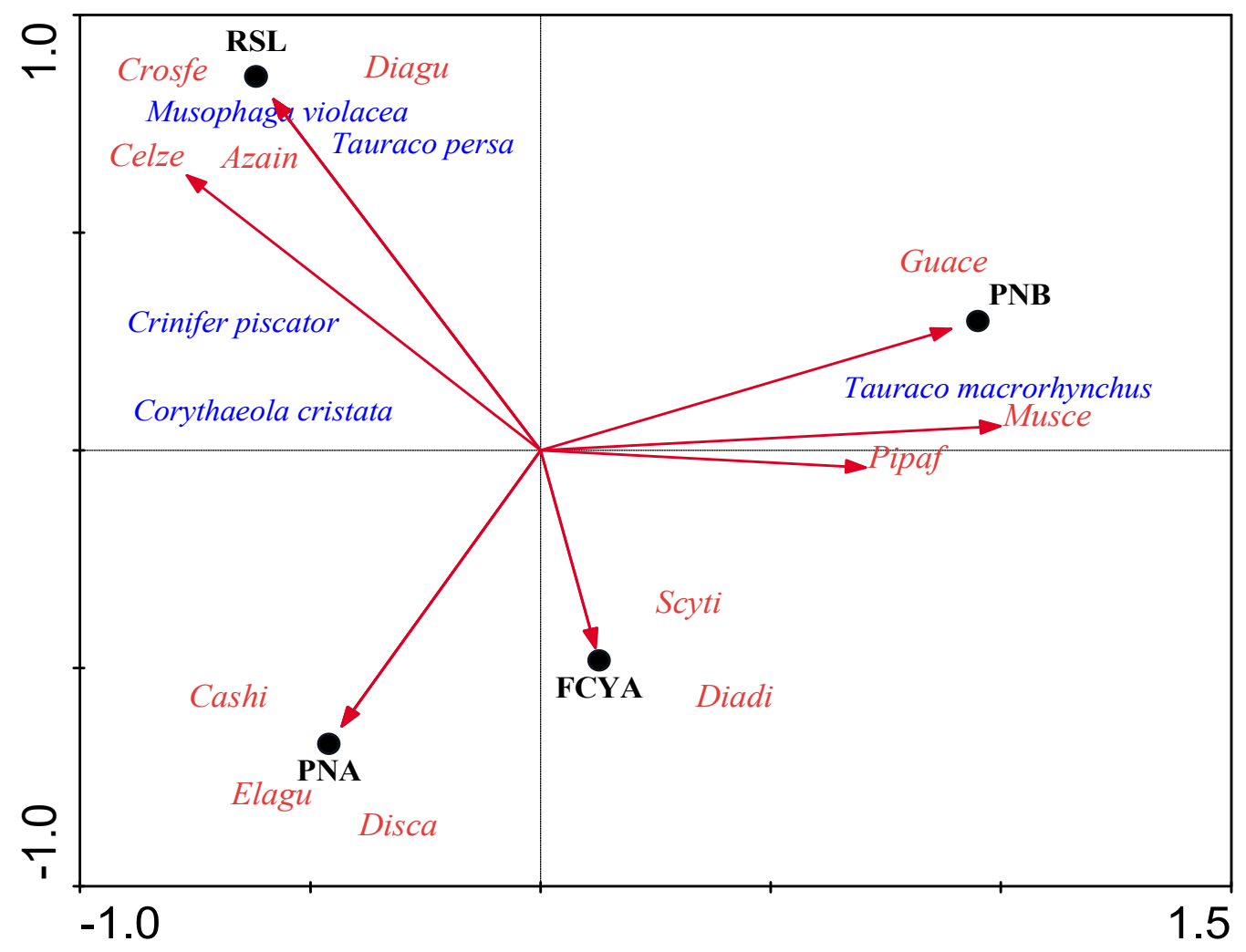

Figure 6: Ordination en RDA des espèces de touracos et des espèces végétales dans l'ensemble des sites Musce: Musanga cecropioides; Guace: Guarea cedrata; Pipaf: Piptadeniastrum africanum; Scyti: Scytopetalum tieghemii; Diadi: Dialium dinklagei; Cashi: Cassipourea hiotou; Disca: Discoglypremna caloneura; Celze: Celtis zenkeri; Azain: Azadirachta indica; Crosfe: Crossopteryx febrifuga; Diagu: Dialium guineense; Elagu: Elaeis guinensis

\section{DISCUSSION}

Au total cinq espèces de touracos ont été inventoriées dans l'ensemble des sites. Leur abondance est élevée pendant la grande saison des pluies (GSP) et la petite saison sèche (PSS) et faible pendant la grande saison sèche (GSS). Cette variation s'explique selon Yeboah et al. (2008), par le fait que, la grande saison des pluies (GSP) et la petite saison sèche (PSS) correspondent à la période de forte disponibilité de fruits dans les milieux naturels. Alors que la faible abondance des touracos pendant la grande saison sèche (GSS) est liée à une baisse des ressources alimentaires des touracos (Yeboah et al., 2008). Ainsi, la variation de l'abondance des touracos serait-elle liée aux différentes périodes de fructification des espèces végétales qu'ils exploitent. Les touracos consomment principalement des fruits, ce sont donc des espèces frugivores (Sun et al., 1997). Cependant, ils consomment d'autres organes végétaux tels que les feuilles et les bourgeons. Cette diversification de leurs ressources alimentaires s'explique selon Izhaki et Safriel (1989) par la faible teneur des fruits en protéines. Ainsi, la consommation des feuilles leur permettrait-elle de compléter leurs besoins en protéines (Sun, 1995). La distribution des touracos n'est pas liée à une essence végétale. Les touracos sont des espèces généralistes qui exploitent une large gamme d'espèces végétales (Sun et Moermond (1997). Cependant, les touracos sont fortement associés à certaines espèces végétales dont ils apprécient les fruits. Par conséquent, une forte densité de ces espèces végétales dans un milieu donné pourrait conditionner leur présence et par conséquent agir sur leur abondance. Cette préférence alimentaire, selon Candy (1984), contraint les touracos à utiliser sélectivement les ressources alimentaires de leurs milieux ce qui justifie leur déplacement d'un endroit à l'autre à la recherche de sites de nourrissage. Sun et Moermond (1997) dans des travaux réalisés dans la forêt classée de Nyungwe au Rwanda, ont 
également affirmé que les touracos ont des préférences alimentaires et utilisent donc sélectivement les ressources alimentaires de leurs milieux. La variation de l'abondance des touracos selon les saisons pourrait

\section{CONCLUSION}

L'étude a révélé que les espèces de touracos sont fortement tributaires des conditions écologiques de leurs milieux. Les touracos ont exploité une large gamme d'espèces végétales. Par ailleurs, leur abondance dans les milieux naturels a variée au cours des saisons. Leur distribution est liée à la disponibilité de ressources alimentaires dans leurs milieux naturels.

\section{REFERENCES BIBLIOGRAPHIQUES}

Bibby C, Jones M, Marsden S, 1988. Expedition Field technique: Bird surveys. Expedition advisory centre, London.

Borghesio L, 1997. Field observations on Prince Ruspoli's Turaco Tauraco ruspolii. Scopus 19: 83-91.

Borrow N et Demey R, 2012. Guide des oiseaux de l'Afrique de l'ouest. Delachaux et Nestlé, Paris, $508 p$.

Candy M, 1984. Habits and breeding biology of the Great blue turaco Corythaeola cristata. Journal of East African Natural History Society, 190: 119.

Demey R et Fishpool LDC, 1991. Additions and annotations to the Avifauna of Côte d'Ivoire. Malimbus, 12: 61-86.

Demey R et Fishpool L D C, 1994. The birds of Yapo Forest, Ivory Coast. Malimbus, 16: 99-114.

Deniveau J L, 1976. Principales caractéristiques physionomiques et floristiques des formations forestières de Lamto (Moyenne Côte d'Ivoire). Ann. Univ. Abidjan, E, 9:26-33.

Gnagbo A, 2016. Diversité des épiphytes vasculaires de la strate inférieure des formations végétales du Parc National d'Azagny (Sud de la Côte d'Ivoire). Journal of Animal \& Plant Sciences 28, (1): 4366-4388.

Izhaki I et Safriel U N, 1989. Why are these so few exclusively frugivorous birds? Experiments on fruit digestibility. Okois, 54: 23-32.

Keitt TH, Bjornstad O N, Dixon P M, Citron-Pousty S, 2002. Accounting for spatial pattern when modeling organism-environment interactions. Ecography, 25: 616-625.

Kouadio K P, Yaokokoré-Beibro K H, Odoukpé KSG, Konan EM, Kouassi KP, 2014. Diversité être liée à ce déplacement. La disponibilité des ressources alimentaires influence donc la distribution des touracos dans les milieux naturels.

Cette étude contribue donc à une meilleure connaissance de l'écologie alimentaire des espèces de touracos. Aussi, dans le cadre de reboisement de conservation des milieux naturels, il conviendrait d'utiliser des espèces végétales locales pour préserver les ressources alimentaires des touracos en vue de leur conservation.

avifaunique du Parc National du Banco, Sudest Côte d'Ivoire. European journal of scientific research, 125(2): 384-398.

Lauginie $F, 2007$. Conservation de la nature et des aires protégées en Côte d'Ivoire. CEDA/NEI, Abidjan, 667p.

Mbété P, Ngokaka C, Akouango F, Bonazebi N, Vouidibio J, 2010. Evaluation des quantités de gibiers prélevées autour du Parc National d'Odzala-Kokoua et leurs impacts sur la dégradation de la biodiversité Journal of Animal \& Plant Sciences. 8 (3): 1061-1069

SODEFOR, 1999. Plan d'aménagement de la forêt classée de Yapo-Abbé 1999-2023 Sodefor édition, 141p.

SODEXAM, 2013. Données météorologiques de la Région de l'Agnéby-Tiassa

Sun C et Moermond TC, 1997. Foraging ecology of three sympatric turacos in a montane forest in Rwanda. The Auk, 114 (3): 396-404.

Sun C, Moermond TC, Givnish TJ, 1997. Nutritional determinants of diet in three turacos in a tropical montane forest in Rwanda. The Auk, 114 (2): 200-211.

Sun C, 1995. Foraging ecology of three sympatric turacos (Musophagidae) and the phenology in an Afro-montane forest. PhD. Dissertation, University of Wisconsin, $182 \mathrm{p}$.

Thiollay J M, 1985. The birds of Ivory Coast: status and distribution. Malimbus, 7. 1-59.

Yaokokoré-Beibro $\mathrm{K} \mathrm{H}$, 2001. Avifaune des forêts classées de l'Est de la Côte d'Ivoire: Données sur l'écologie des espèces et effet de la déforestation sur les peuplements cas des forêts classées de la Béki et de la Bossématié 
(Abengourou). Thèse de Doctorat, Université de Cocody, 245p.

Yeboah S, Deikumah J P, Henaku-Owusu E, 2008. The status of three species of turacos in the Kakum Conservation Area in the Central Region, Ghana. West African journal of Applied Ecology. (13) 104-110. 